\title{
A Micropower Tilt Processing Circuit
}

\author{
Timothy G. Constandinou* ${ }^{* \dagger}$ and Julius Georgiou* \\ *Holistic Electronics Research Lab, ECE Department, University of Cyprus, Nicosia 1678, Cyprus \\ ${ }^{\dagger}$ Institute of Biomedical Engineering, Imperial College London, SW7 2AZ, United Kingdom \\ Email: t.constandinou@imperial.ac.uk, julio@ucy.ac.cy
}

\begin{abstract}
This paper describes a novel analogue circuit for extracting the tilt angle from the output of a standard MEMS accelerometer. The circuit uses the accelerometer signal together with the gravitational acceleration vector to generate the tilt signal. Using a current-mode representation with devices operated in subthreshold, the appropriate trigonometric function has been realised to compute tilt. Furthermore, implementing a long-time constant filter to extract the mean tilt level provides adaptation to the static tilt level. Specifically, this circuit has been designed as part of an implantable vestibular prosthesis to provide inclination signals for bypassing dysfunctional otolith end-organs. The hardware has been implemented in AMS $0.35 \mu \mathrm{m}$ 2P4M CMOS technology.
\end{abstract}

Index Terms - tilt, inclination, trigonometric function, arcsin, vestibular prosthesis, analogue signal processing

\section{INTRODUCTION}

An increasing number of medical devices including implantable prosthetics and body worn instrumentation are incorporating sense systems within and around the body. Physical constraints demand such systems to be compact and lightweight, and the need for autonomy imposes stringent power budgets on such systems. One such sensor is the inclinometer (or tilt sensor), which senses its orientation with respect to gravity.

Tilt sensors have typically implemented using hybrid structures incorporating a stationary reference and movable mass. Traditionally these have been realised using electrolytic fluidfilled cavities, for example- a container in which are patterned an array of incremental electrodes filled with mercury (or nontoxic alternatives). More recently a breed of modern siliconbased inclinometers have emerged implemented in various MEMS technologies [1]-[6]. Although these typically rely on a proof mass being deflected, alternative approaches have been reported using convection [5] and optical micro-filters [6]. It has also been shown how to derive inclination using an unmodified accelerometer [7]-[9].

In this paper, we present a novel hardware implementation of a front end circuit to interface to a MEMS-based capacitive accelerometer and generate a tilt signal. This has been implemented as part of a vestibular prosthesis to provide cues about inclination and bypass dysfunctional otolith end-organs within the vestibule in the inner ear. This paper describes the concept and system architecture (Section II), circuit implementation (Section III) and simulated results (Section IV).

\section{System OVERVIEW}

The circuit presented is this paper is based on the concept that for any sensed acceleration there exists a component due

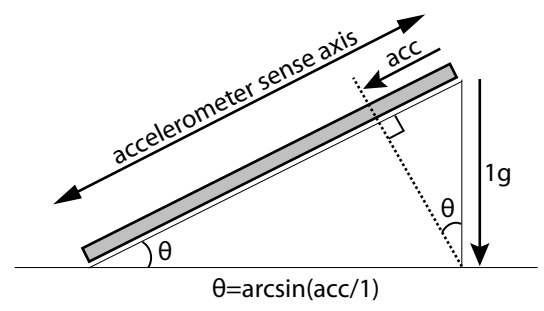

Fig. 1. The concept of obtaining tilt from an accelerometer using the gravitational acceleration vector.

to the gravity, except in the case that the axis of reference is perpendicular to the gravitational acceleration vector. This concept is illustrated in Fig. 1. Consequently, this means that any accelerometer can be used as a tilt sensor provided the following criteria are met: (1) the accelerometers range is equal to or greater than the gravitational constant i.e. $\pm \mathrm{g}$ and (2) the accelerometer does not exhibit any out-of-plane non-linearities, i.e. due to proof mass sagging. However most MEMS-based capacitive accelerometers fulfil this criteria as they are typically designed to be stiff in the out-of-plane axis.

The system architecture of the proposed front-end is shown in Fig. 2. This has been designed to either interface directly to a capacitive accelerometer with capacitance range $\pm 10 \mathrm{pF}$ (corresponding to an acceleration of $\pm 1 \mathrm{~g}$ ), or be used with an off-the-shelf accelerometer taking a voltage output centred between the supply rails. Specifically the Analog Devices ADXL330 has a nominal output (i.e. at $0 \mathrm{~g}$ ) of $0.5 \mathrm{VDD}$ and sensitivity of $0.1 \mathrm{VDD} / \mathrm{g}$. For a $3.3 \mathrm{~V}$ supply this translates to an input range between $1.32 \mathrm{~V}(-1 \mathrm{~g})$ and $1.98 \mathrm{~V}(+1 \mathrm{~g})$ which corresponds to a tilt of between $-90^{\circ}$ and $+90^{\circ}$.

To counteract process variation in the ratiometric output of the accelerometer a long time constant low-pass filter based on an operational transconductance amplifier-capacitor (OTAC) (i.e. GM1 and C) realisation has been used to extract the DC (i.e. for zero tilt) operating point. This assumes the target object will be changing tilt at a higher rate and will inherently provide adaptation to a change in static inclination. For our application this is crucial as this mimics the biomechanical and neural adaptation within the physiological vestibular organ. Alternatively this filter can be replaced with a DAC or voltage reference if an absolute measure of tilt is required about a static reference.

The voltage signals (accelerometer output and extracted DC level) are then converted to a differential current output using a 


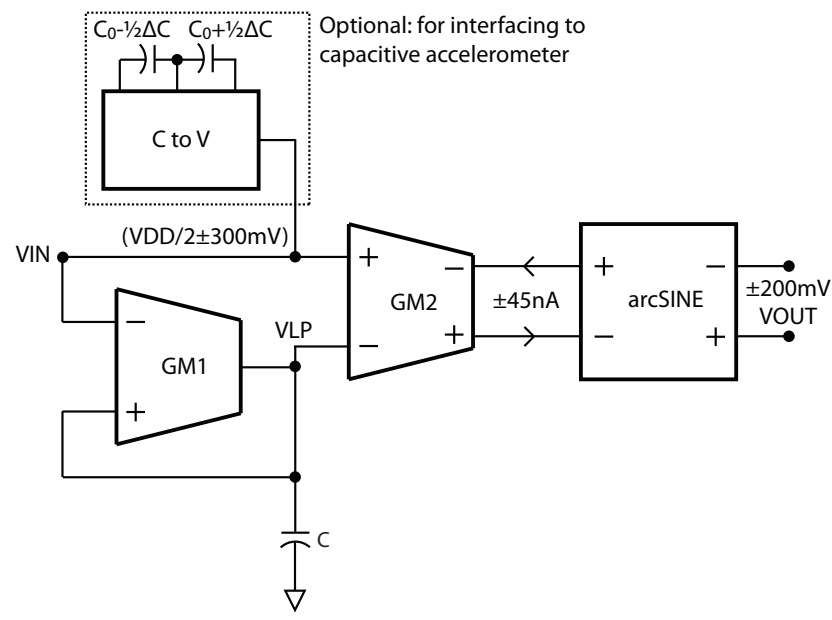

Fig. 2. System architecture for the implementation of the tilt processor concept.

linearised transconductor. This allows for the implementation of a transimpedance arcSINE transfer characteristic.

\section{CiRCUit IMPLEMENTATION}

\section{A. Capacitive Accelerometer Interface}

The sensor interface for a differential capacitive accelerometer is shown in Fig. 3. This consists of a switched capacitor charge integrator to measure a differential capacitance in the range $0-10 \mathrm{pF}$, with $50 \mathrm{fF}$ target resolution. To match the output characteristics of the Analog Devices ADXL330, the following parameters were used: Vref $=200 \mathrm{mV}, \mathrm{C}_{0}=24.27 \mathrm{pF}$. The circuit operates as follows: During $\phi 1$, the output capacitor is reset and differential voltage applied across the input capacitors. During $\phi 2$, the differential voltage applied across input capacitors is inverted and the differential charge difference is converted to a voltage across the op-amp feedback capacitor $\left(C_{F B}\right)$. The input/output characteristics (i.e. capacitance to voltage relationship) is given by:

$$
V_{\text {out }}=\frac{V_{\text {ref }} \cdot \Delta C}{C_{F B}}
$$

\section{B. Low-pass OTA-C filter for DC level extraction}

To extract the DC-level of the accelerometer output, a long time constant filter $(\tau>10 \mathrm{~s})$ has been implemented using an OTA-C implementation. The schematic for the OTA is shown in Fig. 4.

The adopted implementation is based on a balanced OTA and has a 10nA split tail current feeding two PMOS differential pairs Q10-Q11 and Q12-Q13 which share the signal current in a ratio of 1:4. Furthermore, the effective differential pair has been actively linearised by Q8 and Q9, as the required linear input range and current magnitudes made resistive degeneration an unviable option. The current is further scaled in the NMOS current mirrors Q14-17 and Q18-Q21 by a ratio of $5: 1$. Cascoded current mirrors were used to ensure a well-balanced output and all the inner mirrors (i.e. Q6-Q7,

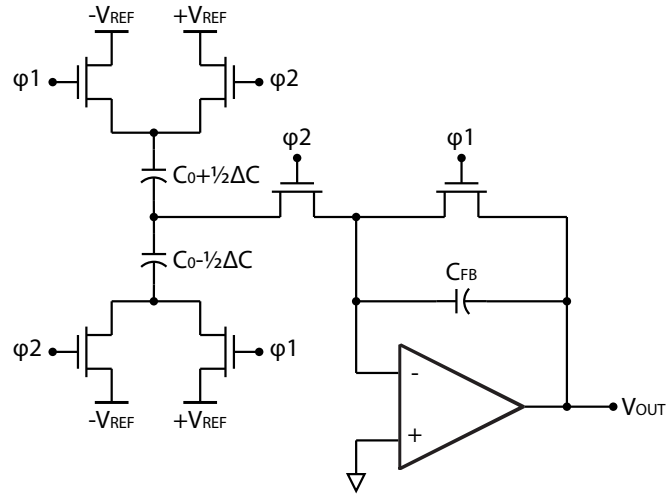

Fig. 3. Circuit schematic of the MEMS accelerometer interface circuit based on a switched-capacitor charge integrator. All switches implemented as transmission gates with $\mathrm{W} / \mathrm{L}=1 \mu \mathrm{m} / 0.5 \mu \mathrm{m}$. This circuit is terminated with a simple sample and hold circuit (not shown).

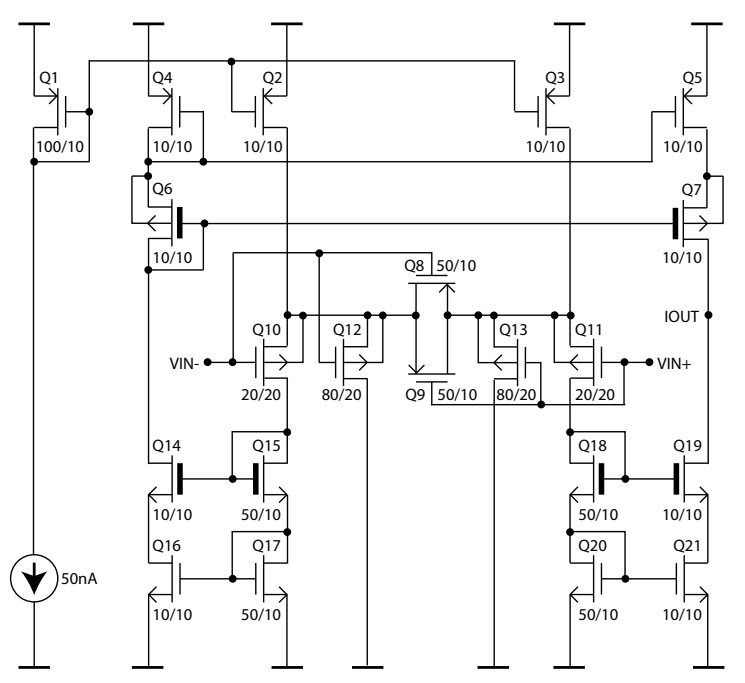

Fig. 4. Circuit schematic of the ultra-low GM operational transconductance amplifier (OTA) used to form a OTA-C low pass filter.

Q14-Q15 and Q18-Q19) have been realised using thick oxide devices. This has been engineered specifically to limit the drain-bulk leakage at low current levels $(<20 \mathrm{pA})$ especially within the standard NMOS devices. All devices have been selected to have relatively long channel lengths $(\mathrm{L}=10 \mu \mathrm{m})$ to improve noise performance (i.e. reducing both flicker and thermal noise contributions) and to achieve good device pair matching.

The achieved transconductance is $18 \mathrm{pS}$ and this coupled with a load capacitance of $40 \mathrm{pF}$, provides a low pass characteristic with time constant of approximately $14 \mathrm{~s}$.

\section{Linearised OTA}

The OTA used to obtain a fully differential current output to drive the arcSINE system is shown in Fig. 5.

This uses a fully differential topology using resistive degeneration to achieve a linearised response for an input range of $\pm 330 \mathrm{mV}$. The circuit feeds a split tail current of $667 \mathrm{nA}$ 


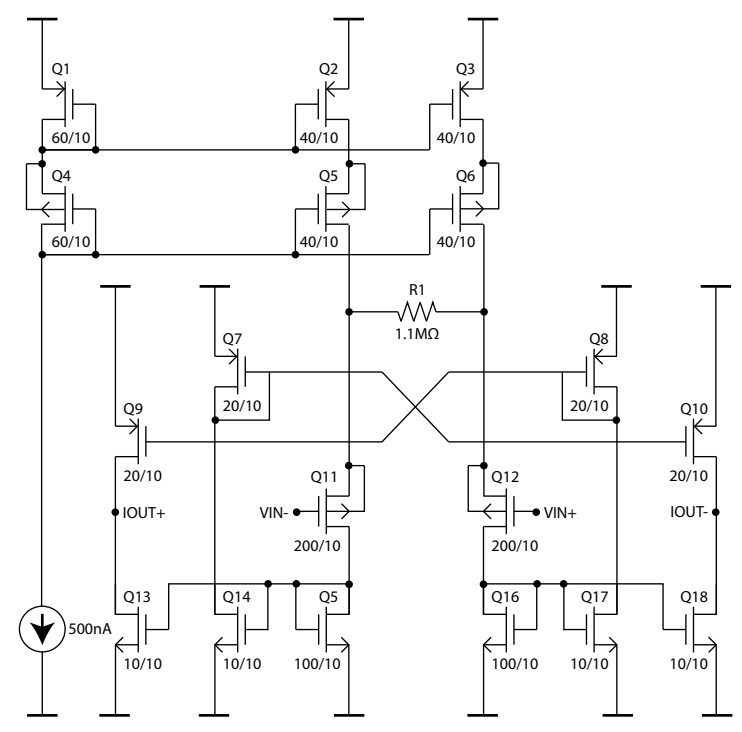

Fig. 5. Circuit schematic of the linearised OTA used to facilitate a fullydifferential $\mathrm{V}$ to I conversion.

into the differential pair (Q11-Q12) and using scaled current mirrors at a 10:1 ratio, an output range of $\pm 45 \mathrm{nA}$ is achieved for the linear input range.

\section{D. arcSINE system}

It has been shown possible to generate a sinusoidal transfer characteristic based on an exponential primitive. Meyer et al. [10] were amongst the first to report that a differential pair with emitter degeneration could be used as a triangle-sine wave converter in bipolar technology. Gilbert [11] extended this technique to provide realisations of other trigonometric functions including their inverses. With weak inversion CMOS technology it has been shown possible to achieve the sine function [12]. However, to use this technique to realise the inverse function would require driving the differential pair with a current and reading out the voltage at the input node.

The implemented circuit producing an arcSINE transfer characteristic is shown in Fig 6. This is based on a weak inversion MOS differential pair which has been diode connected to have a logarithmic input current to output voltage transfer. The emitter degeneration has been implemented in the form of the preceding $\mathrm{V}$ to I conversion (linearised GM detailed previously).

\section{Simulation Results}

The circuit was simulated using the Cadence Spectre (5.1.41isr1) simulator with foundry supplied BSIM3v3 models.

The AC response of the OTA-C low pass filter is shown in Fig. 7. This shows that the time constant of the filter is $13.9 \mathrm{~s}$ (i.e. $\mathrm{F} 3 \mathrm{~dB}=72 \mathrm{mHz}$ ).

Transient simulations taking a static DC operating point and a triangular wave as inputs illustrate the linearity of the transconductor used for the V-to-I are shown in Fig. 8. For input voltages of $\mathrm{V}-=1.65 \mathrm{~V}, 1.32 \mathrm{~V} \leq \mathrm{V}+\leq 1.98 \mathrm{~V}$, the output

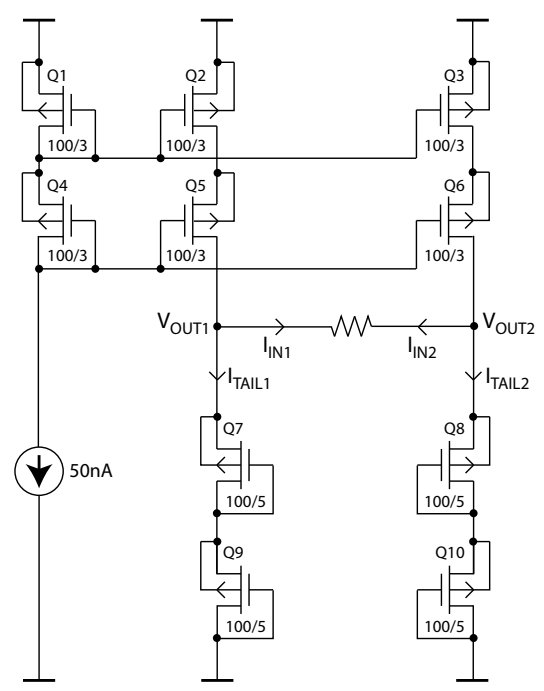

Fig. 6. Circuit schematic of the circuit used to achieve the arcSINE transfer characteristic. This requires a differential input current to provide the resistive degeneration.



Fig. 7. Simulated AC response of the OTA-C low pass filter showing magnitude (top) and phase (bottom) response.

currents are $-45 \mathrm{nA} \leq$ Iout $(-/+) \leq 45 \mathrm{nA}$. This gives a transconductance of $136.4 \mathrm{nA} / \mathrm{V}$.

The arcSINE transfer function has been simulated by providing a differential sinusoidal current to the circuit and observing the differential voltage output. The transient simulation results are shown in Fig. 9. It can be seen that for a differential input current of amplitude $45 \mathrm{nA}$ (frequency $=250 \mathrm{~Hz}$ ), the differential output is a triangular wave of $\pm 225 \mathrm{mV}$ amplitude. Furthermore, the error of the output from an ideal triangle wave is observed to be below $5 \%$.

The design specifications for this system are summarised in Table I.

\section{CONCLUSion}

In this paper we have presented an integrated circuit for use in generating a tilt signal from an accelerometer output. The system designed can either take a voltage input or alternatively interface directly to a MEMS-based capacitive accelerometer. 




Fig. 8. Simulated transient response for the linearised transconductor. Shown is: (a) input voltages ( $\mathrm{V}$ - and $\mathrm{V}+$ ), (b) output currents (I-, I+ and Idiff), and (c) parametric analysis showing effect of resistive emitter degeneration (for $0 \leq \mathrm{R} \leq 1.1 \mathrm{M} \Omega$ ).



Fig. 9. Simulated transient response for the arcSINE transfer function. Shown are (from top to bottom): (a) input currents, (b) output voltages, (c) differential output voltage, and (d) output error.

We have aimed to produce a fully analogue implementation for applications requiring an analogue tilt signal. Furthermore, the circuit extracts the DC operating point through means of a long time constant low pass filter, alleviating the need for calibration or tuning. This additionally provides adaptation to a non-static reference level which is advantageous in many biomedical applications. The intended application for this circuit is as part of a fully implantable vestibular prosthesis for the restoration of balance.

\section{ACKNOWLEDGMENT}

This work was supported by the Cyprus Research Promotion Foundation (RPF), grant no.: $\Pi \Delta E-0505 / 07$. The authors would like to thank Amir Eftekhar and Kritsapon Leelavattananon for useful discussion.

\section{REFERENCES}

[1] R. A. Yotter, R. R. Baxter, S. Ohno, S. D. Hawley, and D. M. Wilson, "On a Micromachined Fluidic Inclinometer," Proceedings of IEEE International Conference on Transducers, Solid-State Sensors, Actuators and Microsystems, vol. 2, pp. 1279-1282, 2003.
TABLE I

SYSTEM PROPERTIES AND TARGET PERFORMANCE

\begin{tabular}{ll}
\hline Technology & AMS $0.35 \mu m$ 2P4M CMOS \\
Supply voltage & $3.3 \mathrm{~V}$ \\
Device count & 152 \\
Low-pass filter bandwidth & $-3 \mathrm{~dB} @ \mathrm{~F}=72 \mathrm{mHz}$ \\
Input voltage range & $1.32 \mathrm{~V}$ to $1.98 \mathrm{~V}$ \\
Output voltage range & $\pm 225 \mathrm{mV}$ (differential signal) \\
Power consumption & $8.46 \mu \mathrm{W}$ \\
\hline
\end{tabular}

[2] K.-S. Kang, H. Jung, D.-S. Kim, B.-J. Kwon, C. J. Kim W.-J. Kim, S.-Y Choi, J.-H. Lee, J.-K. Shin, and S. H. Kong, "Design and fabrication of a MEMS-based electrolytic tilt sensor," Proceedings of the IEEE Conference on Microprocesses and Nanotechnology, pp. 216-217, 2005.

[3] L. Zhao and E. M. Yeatman, "Micro Capacitive Tilt Sensor for Human Body Movement Detection," 4th International Workshop on Wearable and Implantable Body Sensor Networks (BSN 2007), vol. 13, pp. 195200, 2007.

[4] T. G. Constandinou and J. Georgiou, "Micro-Optoelectromechanical Tilt Sensor," Journal of Sensors, vol. 2008, no. 782764, pp. 1-7, 2008

[5] S. Billat, H. Glosch, M. Kunze, F. Hedrich, J. Frech, J. Auber, and W. Lang, "Convection-based Micromachined Inclinometer Using SOI Technology," Proceedings of IEEE International Conference on Micro Electro Mechanical Systems, pp. 159-161, 2001.

[6] N. C. Tien, "Micro-optical Inertial Sensors using Silicon MEMS," Proceedings of IEEE Aerospace Conference, vol. 1, pp. 437-443, 1998

[7] D. Lapadatu, S. Habibi, B. Reppen, G. Salomonsen, and T. Kvisteroy, "Dual-axes capacitive inclinometer/low-g accelerometer for automotive applications," IEEE International Conference on Micro Electro Mechanical Systems, vol. 39, pp. 34-37, 2001.

[8] S. Luczak, et al., "Sensing Tilt With MEMS Accelerometers," IEEE Sensors Journal, vol. 6, no. 6, pp. 1669-1675, 2006.

[9] G. Qinglei, L. Huawei, M. Shifu and H. Jian, "Design of a Plane Inclinometer Based on MEMS Accelerometer," Proceedings of the 2007 International Conference on Information Acquisition, pp. 320-323, 2007.

[10] R. G. Meyer, W. M. C. Sansen, S. Lui and S. Peeters, "The Differential Pair as a Triangle-Sine Wave Converter," IEEE Journal of Solid-State Circuits, vol. 11, no. 3, pp. 418-420, 1976.

[11] B. Gilbert, "A Monolithic Microsystems for Analog Synthesis of Trigonometric Functions and Their Inverses," IEEE Journal of SolidState Circuits, vol. 17, no. 6, pp. 1179-1191, 1982.

[12] O. Ishizuka, Z. Tang and H. Matsumoto, "MOS Sine Function Generator Using Exponential-Law Technique," Electronics Letters, vol. 27, no. 21 , pp. 1937-1939, 1991 